\title{
Screening for impact of popular herbs improving mental abilities on the transcriptional level of brain transporters
}

\author{
PRZEMYSLAW M. MROZIKIEWICZ $Z^{1,2+}$ \\ ANNA BOGACZ ${ }^{1,2}, *$ \\ JOANNA BARTKOWIAK-WIECZOREK ${ }^{1,2}$ \\ RADOSLAW KUJAWSKI ${ }^{2}$ \\ PRZEMYSLAW L. MIKOLAJCZAK 2,3 \\ MARCIN OZAROWSKI ${ }^{2,4}$ \\ BOGUSLAW CZERNY2,5 \\ BEATA MROZIKIEWICZ-RAKOWSKA ${ }^{6}$ \\ EDMUND GRZESKOWIAK ${ }^{1}$
}

\section{${ }^{1}$ Laboratory of Experimental Pharmacogenetics, Department of Clinical Pharmacy and Biopharmacy, University of Medical Sciences, 14 Marii Magdaleny Street, 61-861 Poznan, Poland \\ ${ }^{2}$ Department of Quality Control of Medicinal Products and Dietary Supplements, Institute of Natural Fibres and Medicinal Plants 27 Libelta Street, 61-707 Poznan, Poland \\ ${ }^{3}$ Department of Pharmacology, University of Medical Sciences, 5a Rokietnicka Street, 60-806 Poznan, Poland}

${ }^{4}$ Departament of Pharmaceutical Botany and Plant Biotechnology, University of Medical Sciences, 14 Marii Magdaleny Street 61-861 Poznan, Poland

${ }^{5}$ Department of General Pharmacology and Pharmacoeconomics, Pomeranian Medical University, Zolnierska 48 Street 70-204 Szczecin, Poland

${ }^{6}$ Department of Gastroenterology and Metabolic Diseases, Medical University of Warsaw, Banacha 1a Street, 02-091 Warsaw, Poland
There are a number of compounds that can modify the activity of ABC (ATP-binding cassette) and SLC (solute carrier) transporters in the blood-brain barrier (BBB). The aim of this study was to investigate the effect of natural and synthetic substances on the expression level of genes encoding transporters present in the BBB (mdr1a, mdr1b, mrp1, mrp2, oatp1a4, oatp1a5 and oatp1c1). Our results showed that verapamil caused the greatest reduction in the mRNA level while other synthetic (piracetam, phenobarbital) and natural (codeine, cyclosporine A, quercetin) substances showed a selective inhibitory effect. Further, the extract from the roots of Panax ginseng C. A. Meyer exhibited a decrease of transcription against selected transporters whereas the extract from Ginkgo biloba L. leaves resulted in an increase of the expression level of tested genes, except for mrp2. Extract from the aerial parts of Hypericum perforatum L. was the only one to cause an increased mRNA level for mdr1 and oatp1c1. These findings suggest that herbs can play an important role in overcoming the BBB and multidrug resistance to pharmacotherapy of brain cancer and mental disorders, based on the activity of selected drug-metabolizing enzymes and transporters located in the BBB.

Keywords: ABC proteins, P-glycoprotein, blood-brain barrier, expression level, herbal substances

\footnotetext{
*Correspondence; e-mail: aniabogacz23@o2.pl; † Dedicated to the memory of Professor Przemyslaw M. Mrozikiewicz.
} 
Treatment of several diseases, especially brain diseases and neurological disorders, remains unexplained and creates many therapeutical problems (1). The major challenge for drug delivery to the brain parenchyma is the presence of the blood-brain barrier (BBB), which limits the access of drugs into this place (2). The BBB is a complex of physiological mechanisms, involving physical and biochemical barriers, which prevent penetration of the many endogenous and exogenous substances that may be toxic to the central nervous system (CNS) from the blood into the brain $(1,3)$. The blood-brain barrier involves transmembrane transporters that transport substrates of brain cells to the vascular lumen vessels allowing selective transport of larger molecules as well as those actively exporting feedstock to blood vessels, leading to reduction of intracellular concentration $(4,5)$.

Active transporters involved in the functioning of the blood-brain barrier and the phenomenon of multidrug resistance (MDR) include P-glycoprotein (P-gp), solute carrier (SLC) family transporters such as OATP1A4, OATP1A5, OATP1C1 and multidrug resistance-associated proteins (MRP), inter alia, Mrp1 and Mrp2. P-gp belongs to the family of proteins characterized by the presence of a highly conserved motif, called the ATP-binding cassette (ABC) (6). SLC transporters including OATP1A4 (SLCO1A4), OATP1A5 (SLCO1A5) and OATP1C1 (SLCO1C1) are expressed in the brain and play an important role in the organic anion transport. The effectiveness of synthetic drugs and different plant bio-active metabolites that affect these transporters is inhibited due to their limited ability to penetrate and rapid process of removing substances from the brain $(7,8)$. The occurrence of such interactions is confirmed by animal models and clinical trials in intestinal epithelial cells for P-glycoprotein (9).

In addition, there are few studies on the effects of inhibitors of herbal origin on the level of expression of transporter gene conditioning, phenomenon of multidrug resistance and the blood brain barrier (10). These studies mainly address the impact of synthetic drugs and herbal substances on the P-gp expression level. Results suggest that certain synthetic substances at higher doses, such as as verapamil, are inhibitors for P-gp. However, their highly adverse side effects such as verapamil's cardiac toxicity prevent their practical application in overcoming the multidrug resistance and the blood-brain barrier $(10,11)$. Therefore, the search for safe P-glycoprotein inhibitors of herbal origin is a promising way, in view of fewer side effects, towards normal tissues in comparison with synthetic drugs.

It has been proven that, depending on their concentration, certain flavonoid compounds (kaempferol, daidzein, quercetin, genistein) inhibit the activity of the P-gp pump by reducing the resistance of selected cell lines to vinblastin (12). Moreover, curcumin (from rhizomes of Curcuma longa, L., Zingiberaceae Lindl.), ginsenosides (from roots of Panax ginseng C.A. Meyer, Araliaceae Juss.), piperine (from fruits of Piper nigrum L., Piperaceae, C. Agardh), tannins (from leaves of Camellia sinensis L. Kuntze, Theaceae D. Don) and silymarin (from fruits of Silybum marianum L. Gaertner, Asteraceae Dum.) may inhibit the activity of P-gp. Bergamotin and quercetin from grapefruit juice also modulate the activity of P-gp $(13,14)$.

Taking into account the growing interest among consumers and patients in preparations of plant origin commonly used in the treatment and prevention of symptomatic neurological disorders, there is a need to clarify their potential impact on the CNS-disorder pharmacotherapy. According to our knowledge, relatively little information is available on the effects of plant extracts on the BBB and gene expression in the brain. In 
the case of MRP1 and MRP2, only a few analyses have been performed and are mainly associated with expression in the liver where the drug is primarily metabolized $(8,13)$.

Therefore, the aim of this study was to demonstrate the effect of the selected synthetic drugs as well as substances of plant origin that may play an important role in blocking the transporter functioning in the blood-brain barrier, such as P-glycoprotein (MDR1), proteins associated with multidrug resistance (MRP1, MRP2), SLC transporters OATP1A4 (SLCO1A4) OATP1A5 (SLCO1A5) and OATP1C1 (SLCO1C1) in an in vivo rat model.

\section{EXPERIMENTAL}

\section{Chemicals}

Verapamil, as a standard chemical compound having inhibitory properties against the relevant transporters, and piracetam were purchased from Sigma-Aldrich Chemical Company (USA). Phenobarbital was purchased from PGF Cefarm-Poznan Sp. z o.o. (Poland).

\section{Natural substances}

Quercetin [quercetin dihydrate (purity $=98 \%(\mathrm{HPLC})$ ], codeine (codeine- $\mathrm{d}_{3}$ solution $1 \mathrm{mg} \mathrm{mL}^{-1}$ in methanol, $99 \%$ ) and cyclosporine A (purity $=98.5 \%$ ) A were purchased from Sigma-Aldrich Chemical Company.

\section{Standardized herbal extracts}

Standardized extracts such as that of aerial parts Hypericum perforatum L. (Hypericaceae, Juss.), roots of Panax ginseng C. A. Meyer (Araliaceae Juss.), leaves of Ginkgo biloba L. (Ginkgoaceae Engl.) were used. These were obtained from Finzelberg GmbH \& Co. KG, Germany). The content of active compounds of selected dry alcoholic extracts was determined using high performance liquid chromatography (HPLC) methods according to the procedures included in European Pharmacopoeia (15). Analysis of dried ethanolic extracts from dried aerial parts of Hypericum perforatum (ethanol $60 \%, V / V$ ) revealed the presence of hypericin $(0.3 \%)$ and hyperforin $(0.61 \%)(13)$, from a dried root of - Panax ginseng (ethanol $60 \%, V / V$ ) the presence of ginsenosides (27.1\%) (14), from leaves of Ginkgo biloba (ethanol $80 \%, V / V$ ) the presence of flavonol glycosides (24.5\%), terpene lactones $(13 \%)$ and ginkgolic acids $\left(5.0 \mathrm{~g} \mathrm{~g} \mathrm{~g}^{-1}\right)(15)$.

\section{Animals and treatment}

The experiment with male Wistar rats (300-450) was performed in accordance with Polish govermental regulations and in agreement with the Local Ethics Committee on the Use of Laboratory Animals in Poznan. Male Wistar rats were housed in plastic cages at the Department of Pharmacology, Poznan University of Medical Sciences. Animals were kept in a climate-controlled room under a 12-h light/dark cycle and had access to a commercial rat chow and tap water ad libitum. They were acclimatized for a few days prior to the experiment. All rats were randomly divided into 9 groups from A to J (8 per 
group), which received standardized herbal extracts, natural and synthetic substances suspended in distilled water for 21 days. Group A was treated with verapamil as a potent inhibitor of transporters $\left(20 \mathrm{mg} \mathrm{kg}^{-1} \mathrm{day}^{-1}\right.$, p. o.) whereas group B received cyclosporin A (5 mg kg-1 day-1, p. o.) having also inhibitory properties against the relevant transporters. The next two groups $(C$ and $D)$ were treated with synthetic substances such as piracetam (200 mg kg-1 day-1, p. o.) and phenobarbital (80 $\mathrm{mg} \mathrm{kg}^{-1} \mathrm{day}^{-1}$, p. o.). Group $\mathrm{E}$ was treated with quercetin $\left(500 \mathrm{mg} \mathrm{kg}^{-1} \mathrm{day}^{-1}\right.$, p. o.) while group $\mathrm{F}$ received codeine (20 mg kg-1 day ${ }^{-1}$, p. o.). Another group (group G) was given the standardized extract from aerial parts of Hypericum perforatum $\left(300 \mathrm{mg} \mathrm{kg}^{-1} \mathrm{day}^{-1}\right.$, p. o.) whereas group $\mathrm{H}$ was treated with the root extract of Panax ginseng $\left(30 \mathrm{mg} \mathrm{kg}^{-1}\right.$ day $^{-1}$, p. o.). Group I was given the leaf extract of Ginkgo biloba (200 $\mathrm{mg} \mathrm{kg}^{-1}$ day $^{-1}$, p. o.) and group J was used as a control and was only fed standard diet. Two hours after the last administration, rats were decapitated. The brain tissue (hippocampus) was immediately frozen in liquid nitrogen and stored at $-80{ }^{\circ} \mathrm{C}$.

\section{RNA extraction and $c D N A$ synthesis}

Total cellular RNA was isolated from the rat hippocampus using TriPure Isolation Reagent (Roche, Germany) according to the manufacturer's protocol. Concentrations and the purity of RNA were determined by measuring the absorbance at 260 and $280 \mathrm{~nm}$ using a spectrophotometer (BioPhotometer Eppendorf, USA). RNA samples were stored at $-80^{\circ} \mathrm{C}$. Complementary DNA was synthesized from $1 \mu \mathrm{g}$ of total RNA in a total volume of $20 \mu \mathrm{L}$ using the Transcriptor cDNA First Strand Synthesis Kit (Roche) and oligo(dT) 18 primer (Roche) according to the manufacturer's protocol. The obtained transcripts were stored at $-20^{\circ} \mathrm{C}$ or used directly for the real-time quantitative reverse transcription polymerase chain reaction (RT-PCR).

\section{Determination of $m R N A$ levels by quantitative real-time}

Real-time PCR (RT-PCR) was carried out using a LightCycler ${ }^{\circledR} 480$ instrument (Roche) and a LightCycler ${ }^{\circledR} 480$ SYBR Green I Master (Roche) according to the manufacturer's protocol. Amplicon size and reaction specificity were confirmed by agarose gel electrophoresis and melting curve analysis. The sequences of the primers for mdr1b, oatp1a4, oatp1a5 and oatp1c1 were designed using the Oligo 6.0 program (National Biosciences, USA). Primers for mrp1 and mrp2 were used according to Sugamo et al. (16) and the primers for mdr1a were designed by Andersson et al. (17). All oligonucleotide sequences were synthesized by TIB Molbiol (Poland) and are summarized in Table I. GAPDH cDNA amplification product was used as a housekeeping gene (endogenous internal standard) for normalization. Thermal cycling conditions for analyzed genes were the following: for GAPDH, 35 cycles of $95^{\circ} \mathrm{C}$ for $20 \mathrm{~s}, 59^{\circ} \mathrm{C}$ for $15 \mathrm{~s}, 72^{\circ} \mathrm{C}$ for $15 \mathrm{~s}$; genes encoding transmembrane transporters, 40 cycles of $95{ }^{\circ} \mathrm{C}$ for $20 \mathrm{~s}, 59^{\circ} \mathrm{C}$ for $15 \mathrm{~s}, 72{ }^{\circ} \mathrm{C}$ for $15 \mathrm{~s}$. RT-PCR reaction was carried out in a $10 \mu \mathrm{L}$ reaction mixture. Each PCR set was monitored by measuring the increase in fluorescence by the binding of SYBR Green I dye to the generated double-stranded cDNA. Standard curves were prepared from the dilution of cDNA and generated from a minimum of four data points. All quantitative PCR reactions were repeated twice. The data were evaluated using the LightCycler ${ }^{\circledR} 480$ Basic Software (Roche Applied Science, Berlin, Germany). 
P. M. Mrozikiewicz et al.: Screening for impact of popular herbs improving mental abilities on the transcriptional level of brain transporters, Acta Pharm. 64 (2014) 223-232.

Table I. Sequences of primers used for the RT-PCR analysis

\begin{tabular}{lllc}
\hline Gene & \multicolumn{1}{c}{$\begin{array}{c}\text { Primer sequence } \\
\text { Forward }\left(5^{\prime} ?^{\prime}\right)\end{array}$} & \multicolumn{1}{c}{$\begin{array}{c}\text { Primer sequence } \\
\text { Reverse }\left(5^{\prime} \text { ? 3') }\right.\end{array}$} & $\begin{array}{c}\text { Product } \\
\text { size } \\
(\mathrm{bp})\end{array}$ \\
\hline Mdr1a & GGC CAC ATG ATC AAG ACG & AAC AAG TTG CTG TTC TGC C & 243 \\
Mdr1b & TGA CGT GAA TGA CGC TGG & CAC ATG GCA GAT GAC AAC C & 207 \\
Mrp1 & ATG CTC AAG TGG ACA TGT TTC G & TCG ATT CTG GAC ATG GAT TCG & 144 \\
Mrp2 & CAG GCA ATG GTG TGT ACG AA A & AGG AGT GCT CGT ATC AGA GTC & 105 \\
Oatp1a5 & TGA CAT CTA TAG TTG GAT TCA & TGA GGA CAA GTT GCT TGT AG & 221 \\
Oatp2 & TCG GTA TCC CCA CAT CTA TAG & GAG GAC ACG TTG CTT GTA GG & 226 \\
Oatp1c1 & GAG CAG GAT GCC TGG TCA T & ACT GGT ATC CAC TTC ACA CT & 221 \\
Oatp1a4 & TCG GTA TCC CCA CAT CTA TAG & GAG GAC ACG TTG CTT GTA GG & 226 \\
GAPDH & GAT GGT GAA GGT CGG TGT G & ATG AAG GGG TCG TTG ATG G & 108 \\
\hline
\end{tabular}

GAPDH - glyceraldehyde-3-phosphate dehydrogenase

\section{Statistical analysis}

The mRNA content of the studied genes was expressed as mean \pm SEM. Experimental data were analyzed using the SPSS 17.0 for Windows software. Mean values were compared by means of the one-way ANOVA test. The value of $p<0.05$ was considered as statistically significant.

\section{RESULTS AND DISCUSSION}

In this study, we analyzed the influence of synthetic and natural substances as well as herbal extracts on the expression level of genes encoding transporters involved in BDB and MDR. Our results showed that administration of verapamil as a potential inhibitor of xenobiotic transporters caused a significant decrease of the expression level in all the examined genes [mdrla (93\%), mdrlb (85\%), mrpl (83\%), mrp2 (99.5\%), oatpla4 $(75 \%)$, oatpla5 (90\%), oatplcl $(67 \%)$ ] compared to the control group (Figs. 1 and 2). Cyclosporin A inhibited the expression level by $67 \%(p<0.0001)$ for Mdr1a, $47 \%(p<$ $0.0001)$ for Mrp1, $75 \%(p<0.001)$ for Mrp2 and not significantly for oatp1a4 (22\%). Administration of codeine and piracetam also resulted in a decrease of the mRNA level in all the examined genes for transporters except for oatp1c1, whereas phenobarbital caused an increase of expression level in all the examined genes except for mrp2 where decrease of $99.5 \%$ was envidenced $(p<0.0001)$. Significant induction was observed for mdr1a (6.56-fold, $p<0.0001)$ and mdr1b (7.4-fold, $p<0.0001)$.

In the case of herbal extracts, we showed that Panax ginseng resulted in a decrease of the mRNA level in the examined genes [mdr1a $(23 \%), \operatorname{mrp} 1(63 \%), \operatorname{mrp} 2(24 \%)$, oatp1a4 (32\%), oatp1a5 (33\%), oatp1c1 (21\%)] except for mdr1b. The average mdr1b mRNA expression was higher by $140 \%(p<0.05)$ in the study group compared to the control 
P. M. Mrozikiewicz et al:: Screening for impact of popular herbs improving mental abilities on the transcriptional level of brain transporters, Acta Pharm. 64 (2014) 223-232.

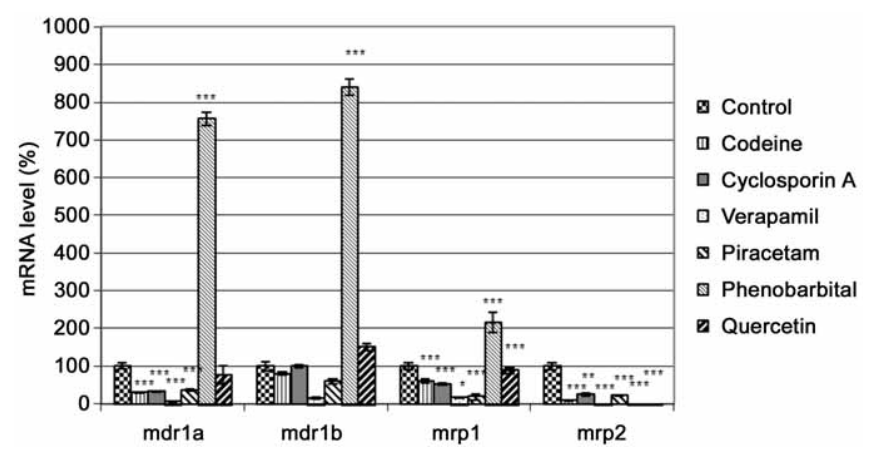

Fig. 1. Effect of synthetic and natural substances on the expression level of genes encoding transmembrane transporters (mdr1a, mdr1b, mrp1 and mrp2) in the rat hippocampus after 21 days of administration. Control groups are defined as $100 \%$. Data are presented as mean \pm SEM $(n=8)$. Asterisks indicate significance (one-way ANOVA test): ${ }^{*} p<0.05,{ }^{* *} p<0.001,{ }^{* * *} p<0.0001$.

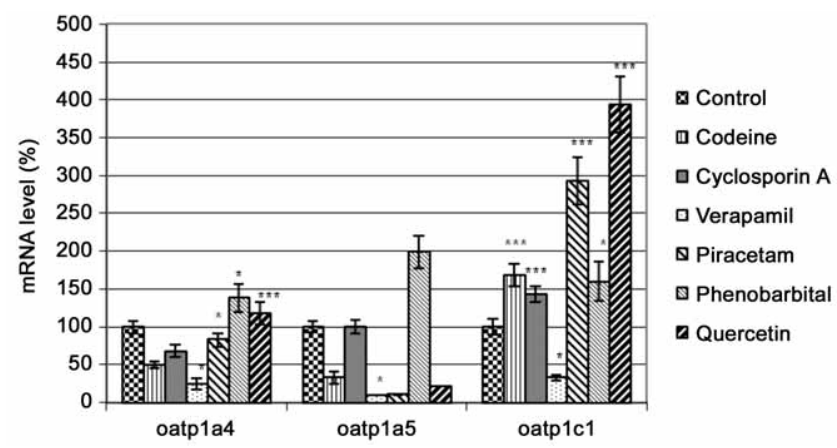

Fig. 2. Effect of synthetic and natural substances on the expression level of genes encoding SLC transporters (oatp1a4, oatp1a5 and oatp1c1) in the rat hippocampus after 21 days of administration. Control groups are defined as $100 \%$. Data are presented as mean $\pm \operatorname{SEM}(n=8)$. Asterisks indicate significance (one-way ANOVA test): ${ }^{*} p<0.05,{ }^{* * *} p<0.0001$.

(Fig. 3). Similar conclusions concerning P-gp were made in the in vivo study conducted by Zhang et al. (18). These authors showed that long-term effects of Panax ginseng caused induction of intestinal and brain endothelium P-glycoprotein expression in rats (18). Another interesting study by Zhang et al. (19) demonstrated that 20(S)-ginsenoside Rh2 from Panax ginseng noncompetitively inhibited P-gp in vitro and in vivo as an example of herb-drug interaction. The in vitro study using rat primary hepatocytes revealed an increase of the mrp2 mRNA level by the addition of red ginseng (100 and $500 \mu \mathrm{g} \mathrm{mL}-1$ ) (20). However, this observation concerned the expression in the liver and not in the brain. Despite the few data on the impact of Panax ginseng on the mRNA level of transporters, our results suggest that the tested extract may have an effective use in overcoming the $\mathrm{BBB}$ or MDR by reducing adverse events in comparison with synthetic drugs. However, 
P. M. Mrozikiewicz et al.: Screening for impact of popular herbs improving mental abilities on the transcriptional level of brain transporters, Acta Pharm. 64 (2014) 223-232.

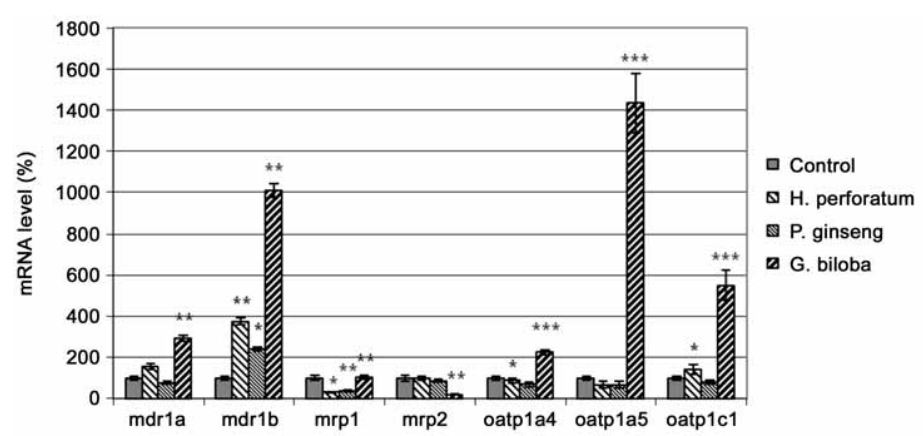

Fig. 3. Influence of standardized herbal extracts on the expression level of genes encoding transmembrane transporters in the rat hippocampus after 21 days of administration. Control groups are defined as $100 \%$. Data are presented as mean \pm SEM of 8 rats in each group. Asterisks indicate significance (one-way ANOVA test): ${ }^{*} p<0.05,{ }^{* *} p<0.001,{ }^{* * *} p<0.0001$.

further studies are needed concerning the influence of Panax ginseng on the transcription of transporter genes, taking into account the duration of extract administration, its dose and composition.

Further, we also analyzed the influence of Hypericum perforatum on the mRNA level in rat hippocampus. Our results showed a noticeable increase of the expression level in the brain tissue by $275 \%(p<0.001)$ for mdr1b and $41 \%(p<0.05)$ for oatp $1 c 1$. A significant mRNA reduction by $68.5 \%$ was observed for mrp1 $(p<0.05)$ (Fig. 3). In the study by Hennessy et al. the authors showed that he Hypericum perforatum extract $(0.15 \%$, $1800 \mathrm{mg}$ in 16 days) may cause an increase of the expression level of the MDR1 gene in the cells of the peripheral blood lymphocytes (PBMCs) even up to 4.2-fold (21). Modulating effect of active compounds of the Hypericum perforatum extract (LI160) on P-gp also confirmed the results of the in vitro study carried out in two cell lines: porcine brain capillary endothelial cells (PBCEC) and VLB-100 (human lymphocytic leukemia cell line) (22). Another interesting study, conducted by Garrovo et al. (23) showed a significant increase in the transcripts for mdrla and mdr1b in all the organs examined (brain, liver, kidney and heart). The increase of mRNA level for mrp2 in the rats treated with St. Johns's wort (100 and $1000 \mathrm{mg} \mathrm{kg}^{-1}$ ) was only observed in the liver and kidney.

In addition, we showed the inductive effect of the standardized Ginkgo biloba extract on the mRNA level of mdr1 (9.1-fold, $p<0.001)$, oatp1a4 (1.25-fold, $p<0.0001)$, oatp1a5 $(13.3$-fold, $p<0.0001)$ and oatp1c1 (4.5-fold, $p<0.0001)$, whereas a decrease of transcription was noted only for mrp2 by $81 \%(p<0.001)$ (Fig. 3). In the study conducted by Li et al. (24), the authors analyzing active substances of Ginkgo biloba showed induction of CYP2B6, CYP3A4, UGT1A1, MDR1, and MRP2 by EGb 761, ginkgolide A (GA) and ginkgolide B (GB), but not by bilobalide (BB) or the flavonoids (quercetin, kaempferol) in human primary hepatocytes. These results suggest that the substances isolated from Ginkgo biloba may act differently in comparison with the whole extract. We believe that the use of Ginkgo biloba extract in the treatment of CNS diseases would be effective only in the case of drugs that are substrates for MRP2. However, these studies need to be confirmed in clinical trials of the effectiveness of Ginkgo biloba in reducing MRP2 gene expression. 
In this study we also analyzed the influence of quercetin on the transcriptional level of tested transporters. The inductive effect of quercetin was observed for mdr1b by $50 \%$ $(p<0.0001)$, oatp1a4 by $18 \%(p<0.0001)$ and oatp1c1 by $293 \%(p<0.0001)$. However, a significant decrease of expression by $99.8 \%(p<0.0001)$ was noted for mrp2 (Figs. 1 and 2). Similar results were obtained by Scambia et al. (25) as well as Shapiro and Ling (26), who showed that quercetin inhibited P-gp. Moreover, it is suggested that low concentrations of quercetin stimulate P-gp. However, high concentrations of this compound inhibit P-gp activity. Explanation of these controversial observations is unknown. In addition, despite similar molecular structures and substrate spectra of MRP1 and MRP2, it was shown that MRP1 has different structural requirements for its inhibition compared to MRP2. Fewer compounds have been shown to be potent inhibitors of MRP2. For example, flavonoids such as apigenin, kaempferol and quercetin may inhibit MRP1 but not MRP2-mediated transport (27). Furthermore, potential in vivo drug interactions due to herbal substances' inhibition of MRP1 and MRP2 is still unclear, because the mechanism of interaction between xenobiotic transporters and substances of plant origin may involve the binding to the nucleotide binding domains and/or the substrate binding site (27). Thus, further studies are needed in order to correctly interpret the animal data and to evaluate the clinical potential for herb-drug interactions.

\section{CONCLUSIONS}

Both the substances of herbal origin and synthetic drugs can modulate the expression of transporters in the blood-brain barrier. Verapamil caused the greatest reduction of the mRNA level of each of the analyzed genes while other synthetic substances showed a selective inhibitory effect. In the case of herbal extracts, it was shown that Panax ginseng can decrease the transcription against selected transporters whereas Ginkgo bil$o b a$ increases the expression level of genes, except for mrp2. Hypericum perforatum only caused an increase of mRNA transcription for mdr1 and oatp1c1. These findings suggest that herbal substances having inhibitory activity against transporters can play an important role in overcoming the BBB and MDR leading to improved brain drug delivery and reduced CNS pathology. In conclusion, the results obtained by our team clearly indicate the validity of the search for potential modulators of the BBB in order to ensure an effective and safe pharmacotherapy of CNS disorders.

Acknowledgments. - This article is dedicated a memory to Professor Przemyslaw M. Mrozikiewicz. This research was supported by the Polish Ministry of Science and Higher Education, Grant No N405 677140.

Acronyms. - ABC - ATP-binding cassette transporters, BBB - blood-brain barrier, CYP - cytochrome P450, CNS - central nervous system, GAPDH - glyceraldehyde 3-phosphate dehydrogenase, MDR - multidrug resistance, MDR1 - multidrug resistance protein 1, MRP1 - multidrug resistance-associated protein 1, MRP2 - multidrug resistance-associated protein 2, OATP - organic anion transporting polypeptide, P-gp - P-glycoprotein, SLC - solute carrier transporters, UGT - UDP-glucuronosyltransferase. 


\section{REFERENCES}

1. N. J. Abbott, Blood-brain barrier structure and function and the challenges for CNS drug delivery, J. Inherit. Metab. Dis. 36 (2013) 437-449; DOI: 10.1007/s10545-013-9608-0.

2. L. A. Khawli and S. Prabhu, Drug delivery across the blood-brain barrier, Mol. Pharm. 10 (2013) 1471-1472; DOI: 10.1021/mp400170b.

3. A. H. Schinkel, J. J. Smith, O. van Tellingen, J. H. Beijnen, E. Wagenaar, L. van Deemter, C. A. Mol, M. A. van der Valk, E. C. Robanus-Maandag and H. P. te Riele, Disruption of the mouse mdr1a P-glycoprotein gene leads to a deficiency in the blood-brain barrier and to increased sensitivity to drugs, Cell 77 (1994) 491-502; DOI: 10.1016/0092-8674(94)90212-7.

4. D. S. Miller, Regulation of P-glycoprotein and other ABC drug transporters at the blood-brain barrier, Trends Pharmacol. Sci. 31 (2010) 246-254; DOI: 10.1016/j.tips.2010.03.003.

5. J. Malmo, A. Sandvig, M. K. Varum and S. P. Strand, Nanoparticle mediated P-glikoprotein silencing for improved drug delivery across the blood-brain barrier: a siRNA-chitosan approach, PLoS ONE 8 (2013) e54182; DOI: 10.1371/journal.pone.0054182.

6. F. J. Sharom, $A B C$ multidrug transporters: structure, function and role in chemoresistance, Pharmacogenomics 9 (2008) 105-127; DOI: 10.2217/14622416.9.1.105.

7. C. J. Bachmeier, W. J. Trickler and D. W. Miller, Comparison of drug efflux transport kinetics in various blood-brain barrier models, Drug Metab. Dispos. 34 (2006) 998-1003; DOI: 10.1124/dmd. 105.006999 .

8. S. P. C. Cole, Targeting multidrug resistance protein 1 (MRP1, ABCC1): past, present, and future, Annu. Rev. Pharmacol. Toxicol. 54 (2014) 95-117; DOI: 10.1146/annurev-pharmtox-011613135959.

9. S. A. Kliewer, The nuclear pregnane $\mathrm{X}$ receptor regulates xenobiotic detoxification, J. Nutr. 133 (2003) 2444S-2447S.

10. B. I. Sikic, Modulation of multidrug resistance: at the threshold, J. Clin. Oncol. 11 (1993) 1629-1635.

11. T. Eichhorn and T. Efferth, P-glycoprotein and its inhibition in tumors by phytochemicals derived from Chinese herbs, J. Ethnopharmacol. 141 (2012) 557-570; DOI: 10.1016/j.jep.2011.08.053.

12. O. Khantamat, W. Chaiwangyen and P. Limtrakul, Screening of flavonoids for their potential inhibitory effect on p-glycoprotein activity in human cervical carcinoma kb cells, Chiang Mai Med. Bull. 43 (2004) 45-56.

13. S. Zhou, L. Y. Lim and B. Chowbay, Herbal modulation of P-glycoprotein, Drug Metab. Rev. 36 (2004) 57-104.

14. P. Limtrakul, O. Khantamat and K. Pintha, Inhibition of P-glycoprotein function and expression by kaempferol and quercetin, J. Chemother. 17 (2005) 86-95; DOI: 10.1179/joc.2005.17.1.86.

15. European Pharmacopoeia, 6th ed., Vol. 2, Council of Europe, Strassbourg Cedex 2008.

16. T. Sugamo, K. Nakamura and H. Tamura, Effects of cytochrome P450 inducers on the gene expression of ocular xenobiotic metabolizing enzymes in rats, J. Health Sci. 55 (2009) 923-929; DOI: 10.1248/jhs.55.923.

17. U. Andersson, K. Grankvist, A. T. Bergenheim, P. Behnam-Motlagh, H. Hedman and R. Henriksson, Rapid induction of long-lasting drug efflux activity in brain vascular endothelial cells but not malignant glioma following irradiation, Med. Oncol. 19 (2002) 1-9.

18. R. Zhang, J. Jie, Y. Zhou, Z. Cao and W. Li, Long-term effects of Panax ginseng on disposition of fexofenadine in rats in vivo, Am. J. Chin. Med. 37 (2009) 657-667; DOI: 10.1142/S0192415X09007144.

19. J. Zhang, F. Zhou, X. Wu, Y. Gu, H. Ai, Y. Zheng, Y. Li, X. Zhang, G. Hao, J. Sun, Y. Peng and G. Wang, 20(S)-ginsenoside Rh2 noncompetitively inhibits P-glycoprotein in vitro and in vivo: a case for herb-drug interactions, Drug Metab. Dispos. 38 (2010) 2179-2187; DOI: 10.1124/dmd. 110.034793 . 
20. A. Kawase, A. Yamada, Y. Gamou, Ch. Tahara, F. Takeshita, K. Murata, H. Matsuda, K. Samukawa and M. Iwaki, Effects of ginsenosides on the expression of cytochrome P450s and transporters involved in cholesterol metabolism, J. Nat. Med. (2013); DOI 10.1007/s11418-013-0791-y.

21. M. Hennessy, D. Kelleher, J. P. Spiers, M. Barry, P. Kavanagh, D. Back, F. Mulcahy and J. Feely, St Johns wort increases expression of P-glycoprotein: implications for drug interactions, Br. J. Clin. Pharmacol. 53 (2002) 75-82; DOI: 10.1046/j.0306-5251.2001.01516.x.

22. C. C. Weber, S. Kressmann, G. Fricker and W. E. Muller, Modulation of P-glycoprotein function by St John's wort extract and its major constituents, Pharmacopsychiatry 37 (2004) 292-298; DOI: $10.1055 / \mathrm{s}-2004-832686$.

23. Ch. Garrovo, A. Rosati, F. Bartoli and G. Decorti, St John's wort modulation and developmental expression of multidrug transporters in the rat, Phytother. Res. 20 (2006) 468-473; DOI: 10.1002/ ptr.1880.

24. L. Li, J. D. Stanton, A. H. Tolson, Y. Luo and H. Wang, Bioactive terpenoids and flavonoids from Ginkgo biloba extract induce the expression of hepatic drug metabolizing enzymes through pregnane $X$ receptor, constitutive androstane receptor, and aryl hydrocarbon receptor-mediated pathways, Pharm. Res. 26 (2009) 872-882; DOI: 10.1007/s11095-008-9788-8.

25. G. Scambia, F. O. Ranelletti, P. B. Panici, R. De Vincenzo, G. Bonanno, G. Ferrandina, M. Piantelli, S. Bussa, C. Rumi, M. Cianfriglia and S. Mancuso, Quercetin potentiates the effect of adriamycin in a multidrug-resistant MCF-7 human breast-cancer cell line: P-glycoprotein as a possible target, Cancer Chemoth. Pharm. 34 (1994) 459-464; DOI: 10.1371/journal.pone.0051764.

26. A. B. Shapiro and V. Ling, Effect of quercetin on Hoechst 33342 transport by purified and reconstituted P-glycoprotein, Biochem. Pharmacol. 53 (1997) 587-596; DOI: 10.1016/S0006-2952(96) 00826-X.

27. M. E. Morris and S. Zhang, Flavonoid-drug interactions: Effects of flavonoids on ABC transporters, Life Sci. 78 (2006) 2116-2130; DOI: 10.1016/j.lfs. 2005.12.003. 